\title{
Forecasting snow avalanches using avalanche activity data obtained through seismic monitoring
}

\author{
A. van Herwijnen ${ }^{\mathrm{a}}$, M. Heck ${ }^{\mathrm{a}}$, J. Schweizer ${ }^{\mathrm{a}}$ \\ ${ }^{a}$ WSL Institute for Snow and Avalanche Research SLF, Davos, Switzerland \\ tel: +41814170161
}

\begin{abstract}
Accurate avalanche occurrence data are of crucial importance for avalanche forecasting, since recent avalanching provides direct evidence on snowpack instability. We therefore explore how avalanche activity data obtained through seismic monitoring can be used for avalanche forecasting. By visually inspecting data from a seismic sensor deployed in an avalanche starting zone, we obtained three avalanche catalogues for two entire winters and one period of 10 days with intense wet-snow avalanche activity. Avalanche activity was clustered in time for all catalogues, and diurnal periodicity was clearly present during spring. In winter, when dry-snow avalanches predominantly release, rather weak long-term correlations on the order of several days were found between past and future avalanche activity. We investigated the performance of a simple model to predict future avalanches based on past avalanche activity. Model performance was better in spring than in winter, especially for very short time scales of up to three hours, and for time scales around 24 hours. Furthermore, the performance of our very simple model was comparable to the performance of more sophisticated models to forecast wet-
\end{abstract}

Email address: vanherwijnen@slf.ch (A. van Herwijnen) 
snow avalanche release based on meteorological input variables. While it is clear that for operational avalanche forecasting automatic avalanche detection still has to be developed, overall this work shows that avalanche activity data obtained through seismic monitoring would yield very valuable data for wet-snow avalanche forecasting.

Keywords: avalanche detection, avalanche forecasting, early warning, seismic monitoring

\section{Introduction}

2 Operational avalanche forecasting mainly relies on meteorological obser3 vations and forecasts in combination with snow cover instability observations, 4 ideally direct observations of avalanches (e.g. McClung and Schaerer, 2006).

5 It involves the assimilation of multiple data sources to make predictions over 6 complex interacting processes. For many decades, avalanche forecasters have 7 developed successful decision-making strategies to deal with this complexity 8 (e.g. LaChapelle, 1980). These rule-based empirical strategies, sometimes 9 applied intuitively, are employed to deal with many of the numerous processes and scales involved in avalanche formation. For instance, avalanche forecasters often rely on the observed or forecasted amount of new snow. However, the success rate of this approach is rather limited, especially for local forecasts (e.g. Schweizer et al., 2009). Employed methods in avalanche therefore prone to subjectiveness.

Monitoring precursor signals to avalanche release could therefore potentially help to improve avalanche forecasting. Acoustic emissions (AE) can 
provide information on the imminence of failure of a material (e.g. Michlmayr et al., 2012), and AE monitoring in laboratory experiments has extensively been used to better understand the failure process in various materials (e.g. Lockner, 1993; Woo et al., 2008; Niemz et al., 2009; Rosti et al., 2010). On the larger scale, this technique has also been used to monitor and study gravitational slope instabilities such as landslides (e.g. Spillmann et al., 2007), cliff collapse (Amitrano et al., 2005), rock avalanches (e.g. Deparis et al., 2008) and unstable glaciers (e.g. Faillettaz et al., 2008). However, despite encouraging new results (Kapil et al., 2014; Reiweger et al., 2015), due to the strong attenuation of high frequency waves in snow, the applicability of AE for forecasting avalanches is likely limited. Furthermore, reliable low frequency precursor signals to avalanche release have not yet been identified (van Herwijnen and Schweizer, 2011a), even though previous studies suggested that such signals exist (Gubler, 1979).

In the absence of reliable, non-invasive, in-situ precursor signals from the snowpack associated with imminent slope instability, avalanche occurrence data might offer an alternative. Indeed, recent avalanche observations, for instance the number of observed avalanches within the last 24 hours, are widely used for avalanche forecasting (e.g. McClung and Schaerer, 2006). During snow storms, the likelihood of increased avalanching is usually obvious, for instance, once about $50 \mathrm{~cm}$ of new snow has fallen. However, due to the absence of reliable avalanche observations, it often remains uncertain whether many natural avalanches occur. The answer can usually only be given after the storm, once visibility has improved, when the danger has already decreased and the peak activity has generally passed. Clearly, more 
reliable methods to detect avalanche occurrence are required.

For the automated remote detection of avalanches several techniques and instruments can been applied, in particular ground based and satellite radar sensors (e.g. Gauer et al., 2007; Kogelnig et al., 2012; Eckerstorfer et al., 2016), infrasonic sensors (e.g. Marchetti et al., 2015; Scott et al., 2007; Thüring et al., 2015), and seismic sensors. The latter are well suited for the remote detection of hazardous mass movements such as rockfall, landslides, ice avalanches and snow avalanches (e.g. Caplan-Auerbach and Huggel, 2007; Deparis et al., 2008; Suriñach et al., 2005). Seismic monitoring systems consist of one or several seismic sensors, also called geophones or seismometers, fixed in the ground or bolted to rocks to record ground vibrations generated by mass movements. Ives et al. (1973) were the first to show that seismic monitoring equipment can be used to remotely detect avalanches. It quickly became evident that such avalanche monitoring systems could potentially provide valuable information for avalanche forecasting, since data on avalanche activity represent the most direct instability data (McClung and Schaerer, 2006). As noted by Harrison (1976): 'a network of a few sensors which remotely could detect and locate avalanches during storm conditions over an area of a few square miles would have important application'. Since then, numerous studies have shown that, depending on the size, avalanches that occur up to several kilometers away from the sensors can be detected (e.g. Bessason et al., 2007; Suriñach et al., 2001; van Herwijnen and Schweizer, 2011a; Lacroix et al., 2012).

Thus far, seismic monitoring systems are not widely used for operational avalanche forecasting, in part due to the difficult task of automatically dis- 
criminating signals generated by avalanches from background noise. Indeed, while Rubin et al. (2012) obtained encouraging detection rates using various machine learning algorithms for the automatic detection of avalanche signals in seismic data, the high false alarm rate of around $90 \%$ showed that substantial progress still has to be made. Nevertheless, based on visual inspection of the data from one seismic sensor, van Herwijnen and Schweizer (2011b) were able to obtain a database with the release times for 385 avalanches in one winter using data from one sensor, with an estimated accuracy of less than one minute. Similarly, Lacroix et al. (2012) visually identified 80 avalanches in the data from a seismic array with seven sensors for one winter. Such avalanche catalogues can be used to better understand avalanche triggering mechanisms and ultimately improve avalanche forecasting, as suggested by recent results presented by van Herwijnen and Schweizer (2011b), Lacroix et al. (2012) and Schweizer and van Herwijnen (2013).

By extending the previously published avalanche data base from van Herwijnen and Schweizer (2011b), in this study we explore how avalanche activity data obtained through seismic monitoring can be used for avalanche forecasting. First we investigate clustering and periodicity in avalanche activity and compare local avalanche activity obtained through seismic monitoring with regional avalanche activity obtained through conventional visual observations. Then, we focus on exploiting avalanche activity data for local avalanche forecasting (about $2 \mathrm{~km}^{2}$ ) at time scales of up to 7 days. To evaluate the effectiveness and reliability of the forecast, we use two methods: receiver operating characteristic curves (e.g. Kumar and Indrayan, 2011) and error diagrams (e.g. Molchan, 1997). These diagrams allow to evaluate fore- 
93

cast performance in terms of probability of detection, false alarm ratio and alarm duration, and have been used to evaluate forecasting models for earthquakes and volcanic eruptions (e.g. Talbi et al., 2013; Grasso and Zaliapin, 2004). Due to a lack of accurate avalanche activity data, such a rigorous performance evaluation is generally not possible in avalanche forecasting.

\section{Methods and data}

\subsection{Sites and instrumentation}

We monitored avalanche activity at two sites above Davos (Figure 1): the Wannengrat (WAN) and the Dorfberg (DFB) field sites. At both sites, we continuously record seismic data, one or several automatic meteorological stations (AMS) are present and we installed automatic cameras which allowed us to identify seismic signals generated by avalanches (van Herwijnen and Schweizer, 2011a,b). The field sites and data acquisition systems are only briefly described; a detailed description can be found in van Herwijnen and Schweizer (2011a), van Herwijnen and Schweizer (2011b) and van Herwijnen et al. (2013).

At the WAN field site, we deployed an array of 7 vertical uni-axial geophones with a natural frequency of $14 \mathrm{~Hz}$ in an avalanche starting zone at an elevation of about 2500 m.a.s. l. The commercial data acquisition system (Seismic Instruments SmartGeophone system) consists of low-noise, high-precision 24-bit analog to digital converters (ADC) and data were continuously sampled at a rate of $500 \mathrm{~Hz}$. The geophones were mounted in a foam housing with a density close to that of snow and placed on top of the snow cover in early winter to be buried by subsequent snowfall (van Herwij- 
nen and Schweizer, 2011a). At the DFB field site, we deployed one vertical component geophone with a natural frequency of $14 \mathrm{~Hz}$ on a grassy slope at an elevation of about 1900 m.a.s.l. The data acquisition system (Cirrus Logic CRD5378) consists of a low-noise, high-precision 24-bit ADC and data were continuously sampled at a rate of $500 \mathrm{~Hz}$. The geophone was placed in a waterproof plastic housing and inserted in the ground with a metal spike.

\subsection{Signal processing and avalanche detection}

Since there is considerable environmental noise in seismic data, for instance due to airplanes, wind or ski lifts, it is important to distinguish seismic signals generated by avalanches from noise (van Herwijnen and Schweizer, 2011b). Using avalanches identified on the images from automatic cameras allowed us to visually identify typical characteristics associated with signals generated by avalanches (see Figures 2 and 3 for examples). This learning step was required for each site separately, since the location of the site as well as local topography and geology strongly affect the seismic wave field. For instance, due to its proximity to the town of Davos and a large ski area (Figure 1), there was substantially more noise in the seismic data at DFB than at WAN (compare the signal-to-noise levels in Figures 2 and 3). Since there was a clear diurnal trend in the signal-to-noise ratio at the Dorfberg field site (not shown), we are confident that the increased noise at this field site is of anthropogenic nature.

Despite these site specific differences, seismic signals generated by avalanches had unique temporal and spectral characteristics allowing us to identify them in the seismic data. To do so, we performed a visual inspection of the waveform and the spectrogram (see Figures 2 and 3 for examples), i.e. the evo- 
lution of the frequency content of the signal with time, as was done by van Herwijnen and Schweizer (2011b) and Lacroix et al. (2012). A visual inspection of the seismic data is time consuming and prone to observer bias. To reduce the latter, all data were inspected by the same observer. However, in the absence of reliable automatic signal classification methods, visual inspection of the data is thus far the only method to identify signals generated by avalanches. While we have invested much effort to obtain validation data from the field (for a detailed description see van Herwijnen and Schweizer, 2011b), many of the avalanche signals identified in the seismic data were not confirmed by field observations (see Figure 4 for an example). For instance, at DFB during the spring of 2013, 25 of the 52 avalanche signals identified in the seismic data remained unconfirmed. Nevertheless, given the overall similarities between the seismic signals generated by avalanches, we are relatively confident in the accuracy of our visual inspection of the data.

\subsection{Seismic avalanche catalogues}

For this study, we analyzed seismic data during three distinct periods: for WAN we inspected data from one sensor inserted in the snow from two winters, namely the winters of 2009-2010 and 2010-2011, and for DFB we focused on a period of high wet-snow avalanche activity during the spring of 2013. We thus have three seismic avalanche catalogues, henceforth referred to as WAN2010, WAN2011 and DFB2013, consisting of the release time $t_{i}$ of each avalanche found in the seismic data. The release time was used to calculate the avalanche activity $N(t, s)$, consisting of the number of avalanches with $t_{i} \in[t-s, t)$ and $s$ the length of the time interval in hours. For instance, for $s=1$ hour we obtain an hourly avalanche activity, while for $s=24$ hours 
we obtain daily avalanche rates. Finally, besides the avalanche activity we also computed the waiting time between subsequent avalanches, defined as $\tau_{i}=t_{i}-t_{i-1}$

WAN2010 consisted of 385 avalanches over a period of 108 days from 13 January to 30 April 2010 (blue line in Figure 5). WAN2011 consisted of 154 avalanches over a period of 138 days from 11 Decemeber 2010 to 27 April 2011 (red line in Figure 5). Finally, DFB2013 consisted of 52 avalanches over a period of 10 days from 1 to 11 March (green line in Figure 5). To investigate differences in dry-snow and wet-snow avalanching, we further split the WAN2010 and WAN2011 catalogues in a winter period, when the majority of avalanches consisted of dry-snow avalanches, and a spring period, when most avalanches were wet-snow avalanches. Based on field observations as well as a detailed inspection of local meteorological data, we determined that for WAN2010 the cutoff date between winter and spring was 15 March 2010, while for WAN2011 it was 25 March 2011.We will refer to these avalanche sub-catalogues as WAN2010 winter, WAN2010 $_{\text {spring, }}$ WAN2011 winter $_{\text {}}$

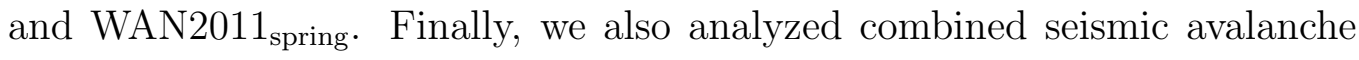
catalogues, namely SEIS consisting of WAN2010, WAN2011 and DFB2013 combined, SEIS $S_{\text {winter }}$ consisting of WAN2010 winter and WAN2011 $1_{\text {winter }}$, and SEIS $_{\text {spring }}$ consisting of WAN2010 spring, WAN2011 $_{\text {spring }}$ and DFB2013.

\subsection{Regional avalanche catalog}

For the region of Davos, the avalanche warning service monitors an area of $175 \mathrm{~km}^{2}$ on a daily basis and information is gathered on the number, size and type (dry or wet-snow) of avalanches. The uncertainty in the time of occurrence can sometimes be large as observations cannot be made during 
periods of bad visibility, for instance during snow storms. However, there are no consistent records of this uncertainty. For this study, we compiled a regional avalanche catalog consisting of all avalanche observations during the study period. Since there is also information on the type of avalanche, we further compiled two additional catalogues consisting of all dry-snow and all wet-snow avalanche observations during the study period. We thus have three regional avalanche catalogues, henceforth refereed to as $\mathrm{OBS}, \mathrm{OBS}_{\mathrm{dry}}$ and $\mathrm{OBS}_{\text {wet }}$, consisting of the estimated release date of each avalanche observed in the region of Davos. We also compared the avalanche catalogues to the forecasted avalanche danger level for the region of Davos, based on the European five-degree danger scale (1: Low, 2: Moderate, 3: Considerable, 4: High, 5: Very High) (e.g. Meister, 1995; Schweizer et al., 2003).

For correlation analysis, e.g. between avalanche activity catalogues, we used the Spearman rank order correlation coefficient $r_{s}$. A level of significance $p=0.05$ was chosen to decide whether the correlations were statistically significant.

\subsection{Model performance measures}

In this work we explore how avalanche activity data obtained through seismic monitoring can be used for forecasting avalanches. We therefore investigated a simple model based on past avalanche activity to predict future avalanches (Section 3.4). To evaluate the predictive performance of our model, we used common performance measures such as the probability of detection (POD) and the false alarm ratio (FAR) (e.g. Wilks, 2011), and in particular two methods: receiver operating characteristic (ROC) curves (e.g. Kumar and Indrayan, 2011) and error (ERR) diagrams (e.g. Molchan, 1997). 
The ROC curve displays the probability of detection (POD; the number of correctly predicted events divided by the total number of events) versus the false positive rate (FPR; the number of falsely predicted events divided by the total number of non-events) for varying avalanche activity thresholds. The area under the curve (AUC) is an effective measure to assess the performance of a model (for more details, see Kumar and Indrayan, 2011). An AUC value of 1 corresponds to a perfect model, while an AUC value of 0.5 corresponds to a flip of a coin (50-50 chance).

Error diagrams allow to characterize model performance in terms of failure to predict rate (1-POD) and total alarm duration (DUR; length of time with avalanche activity above the threshold divided by total length of the time series) for varying avalanche activity thresholds. Error diagrams thus add an important parameter when evaluating model performance, namely the total alarm duration. A DUR value close to 1 corresponds to a poor model since an alarm is raised for most of the time. The optimal avalanche activity threshold is determined by finding the minimum distance:

$$
d_{\text {min }}=\min \left(\sqrt{\left(d_{R O C}^{2}+d_{E R R}^{2}\right)}\right)
$$

with $d_{R O C}^{2}=F P R^{2}+(1-P O D)^{2}$ the distance between a perfect model (point $(0,1))$ and the observed ROC curve, and $d_{E R R}^{2}=(1-P O D)^{2}+D U R^{2}$ the distance between a perfect model (point $(0,0)$ ) and the observed ERR curve. For the optimal activity threshold, we then determined the corresponding POD and DUR values as well as the false alarm ratio (FAR; the number of falsely predicted events divided by the total number of predicted events). Evaluating model performance can thus be done using four distinct metrics: AUC, POD, FAR and DUR. To analyze our avalanche forecasting model in 
a comprehensive manner, we therefore determined the overall model score $S$ defined as $S=(1-A U C)+(1-P O D)+F A R+D U R$. A perfect model would therefore have a score of 0 , while a score of 2 or more would imply poor model performance.

\section{Results and discussion}

Information on the size and type of the avalanches detected in the seismic data was generally not available. For the WAN2010 catalog, most avalanches occurred after the cutoff date between winter and spring (red dashed line in Figure 5), suggesting that the majority of avalanches in this catalog were wetsnow avalanches. For WAN2011, the avalanches were more or less equally split between winter (88) and spring (66) (blue dashed line in Figure 5). Finally, for the DFB2013 catalog, 27 of the 52 avalanches were seen on the images from the automatic cameras. All these were wet-snow avalanches, indicating that the avalanches in this catalog were most probably all wetsnow avalanches.

Based on an analysis of the avalanches observed on the images from the automatic camera, van Herwijnen et al. (2013) determined that avalanche length ranged from 13 to almost 700 meters. Furthermore, they showed that avalanche length was proportional to the square of seismic signal duration T. For WAN2010, van Herwijnen and Schweizer (2011b) showed that many signals identified in the seismic data were generated by wet loose snow avalanches. While some of these loose snow avalanches were relatively small, others entrained the entire snow cover, resulting in larger avalanches which generated relatively long $(T>20 \mathrm{~s}$ ) seismic signals (see Figure 6 and 7 in van 
Herwijnen and Schweizer, 2011b). Furthermore, they showed that the cumulative distribution of $T^{2}$ followed a power law with an exponent of $2.5 \pm 0.4$, similar to the exponent of $2.2 \pm 0.1$ measured for snow slab avalanche scaling based on slab width field measurements (Faillettaz et al., 2004). Overall, results from van Herwijnen et al. (2013) and van Herwijnen and Schweizer (2011b) suggest that $T^{2}$ provides some basic information on avalanche size, albeit indirect. The distributions of $T^{2}$ thus suggest that the DFB2013 catalog contained the largest avalanches, while the WAN2011 catalog contained the smallest avalanches (Figure 6).

\subsection{Clustering in avalanche activity and periodicity}

The hourly avalanche activity observed at our seismic monitoring stations was clustered in time (Figure 5). Indeed, for WAN2010 there were two very distinct peaks in the avalanche activity, around 20 March and at the end of April. Even though avalanche activity was substantially lower for WAN2011, there were also several periods with above average avalanche activity (e.g. early March and early April). Finally, in the much shorter catalog of DFB2013, avalanche activity was highest near the end of the period.

To verify the non-random distribution of avalanche occurrence in more detail, we determined the coefficient of variation $\eta$ of the waiting time $\tau$, defined as the ratio between the standard deviation and the mean of $\tau$ (Marzocchi and Zaccarelli, 2006). For a Poisson process, i.e. a uniform distribution in time, $\tau$ obeys an exponential distribution and $\eta=1$. Clustered events are characterized by $\eta>1$. The assumption that the occurrence times obey a Poisson process was rejected for all catalogues (Table 1), except for WAN2010 ${ }_{\text {winter }}$. 
To further investigate clustering, the cumulative distribution of $\tau$ was analyzed for all seismic avalanche catalogues (Figure 7). Clearly, for all catalogues the waiting times were not uniformly distributed, except for WAN2010 ${ }_{\text {winter }}$. For short time scales, the waiting times were less clustered than a Poisson process, while for longer time scales $\tau$ was more clustered. By comparing the observed cumulative distributions with the expected exponential distribution (dashed lines in Figure 7 ), one can determine a threshold time $t_{r}$ above which larger waiting times are more frequent than expected (Tatard et al., 2010). For WAN2010 and DFB2013 $t_{r}$ was around 12 hours, while for WAN2011 it was more than 40 hours (Figure 7a and Table 1). When analysing the winter and spring catalogues separately, the lowest $t_{r}$ values were found for the spring catalogues (Table 1, Figure 7b and 7c). Overall, these result show that avalanches do not occur randomly. This was expected since avalanches result from the complex interaction between meteorological disturbances (e.g. precipitation or incoming solar radiation) and existing weaknesses within the snow cover, acting over various time scales. Our results suggests that in spring avalanches are clustered for time scales on the order of several hours to one day, while in winter clustering time scales are on the order of a few days.

To investigate periodicity in the avalanche activity, we calculated the covariance of the hourly avalanche activity

$$
C(r, s)=\frac{1}{n} \Sigma_{t=1}^{t=n-r} N(t, s) N(t+r, s)
$$

with $r$ the lag time and $s=1$ hour. The normalized covariance $\frac{C(r)}{C(0)}$ exhibited a first zero crossing after a lag time of 12 hours and a secondary maximum for a lag time of 24 hours, especially for WAN2010 and DFB2013 (Figure 8a). 
This suggests that there generally was a daily pattern in avalanche activity, which comes as no surprise since we mostly detected wet-snow avalanches, which typically release during the day when the energy input into the snow cover is greatest (e.g. Mitterer and Schweizer, 2013; Helbig et al., 2015). This periodicity in wet-snow avalanching is further highlighted when we analyze $\frac{C(r)}{C(0)}$ for winter and spring separately (Figure 8b). For WAN2010 winter,

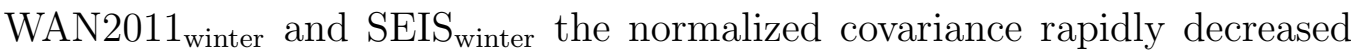
to values close to zero for lag times larger than two hours (Figure 8b), suggesting there was no discernable periodicity in dry-snow avalanche activity.

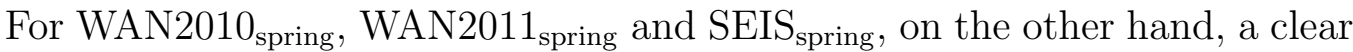
daily pattern emerged with a minimum in $\frac{C(r)}{C(0)}$ after a lag time of 12 hours and a secondary maximum for a lag time of 24 hours (Figure 8c).

\subsection{Comparison with regional avalanche activity and avalanche danger level}

From an avalanche forecasting perspective, observations of recent avalanche occurrences are regarded as unambiguous information on snow cover instability (e.g. McClung and Schaerer, 2006). Avalanche observations are therefore commonly considered as the best predictor for future avalanches. The area monitored by our seismic monitoring stations is approximately $2 \mathrm{~km}^{2}$ (van Herwijnen and Schweizer, 2011b; van Herwijnen et al., 2013). To evaluate how representative the avalanche activity from the seismic sensors is from an avalanche forecasting perspective, we therefore compared daily seismic avalanche activity with the avalanche activity observed in the region of Davos. There was a weak positive correlation between regional avalanche activity recorded through conventional visual observations and avalanche activity recorded through seismic monitoring, although for DFB2013 the corre- 
lation was not significant (Table 2 and Figure 9). These results suggest that increased regional avalanche activity generally also coincided with increased local avalanche activity near the seismic monitoring stations.

Despite the small area covered by the seismic stations, compared to about $175 \mathrm{~km}^{2}$ for the visual observations in the region of Davos, all the major regional wet-snow avalanche periods were well captured (compare solid red and dashed green lines in Figure 9). This is also reflected by the significant positive correlation between the observed wet-snow avalanche activity and seismic avalanche activity, except again for DFB2013. While for DFB2013 the overall pattern in avalanche activity corresponded well with observed wet-snow avalanches, the absence of a significant correlation was due to the fact that the peak in avalanche activity at the seismic station was on 8 March, while it was a day later for the regional observations (Figure 9c). Indeed, when shifting the visual observations of regional avalanche activity backward by one day, the correlation became very strong and significant for all observed avalanches $\left(r_{s}=0.88, p<10^{-3}\right)$ as well as for the wet-snow avalanches $\left(r_{s}=0.86, p<10^{-3}\right)$. Finally, there was a major avalanche cycle at the end of April 2010 in the WAN2010 catalog which was not as prevalent in the $\mathrm{OBS}_{\text {wet }}$ catalog. The reason for this is unknown, even though in general avalanche observers tend to underreport avalanches this late in the season, especially smaller wet-snow avalanches.

Dry-snow avalanche cycles were generally less well captured through seismic monitoring, with the exception of an avalanche cycle in March 2011 (Figure 9b), as reflected by a lack of correlation between $\mathrm{OBS}_{\mathrm{dry}}$ and seismic avalanche activity (Table 2). These results suggest that wet-snow avalanches 
generate larger seismic signals than dry-snow avalanches of comparable size, as previously reported (Biescas et al., 2003; van Herwijnen and Schweizer, 2011a). Furthermore, our results suggest that monitoring a relatively small area with one seismic station can provide reasonable estimates for regional wet-snow avalanche activity. For dry-snow avalanches, on the other hand, monitoring a larger area by deploying more seismic stations is likely required to estimate regional activity.

Regional avalanche activity data obtained through conventional visual observations are widely used in avalanche forecasting. For the region of Davos, it therefore comes as no surprise that there was a significant positive correlation between forecasted avalanche danger level and observed regional avalanche activity from the previous day (Table 3). While this was the case for both dry-snow and wet-snow avalanche danger, the correlation was strongest for dry-snow avalanche danger (Table 3 and Figure 10a). Similar to regional avalanche observations, local daily avalanche activity observed through seismic monitoring generally increased with increasing forecasted avalanche danger level, even though the correlation was somewhat weaker (Table 3). However, when looking at dry-snow and wet-snow avalanche danger separately, a correlation only existed for wet-snow avalanche danger (Table 3 and Figure 10b). Overall, this suggests that local avalanche activity data obtained through seismic monitoring could yield valuable data to estimate the regional avalanche danger level, in particular for wet-snow avalanches.

\subsection{Correlation of past and future avalanche activity}

The observed correlation between avalanche activity and forecasted avalanche danger level does not necessarily imply that past avalanche activity is in- 
dicative of future activity. To investigate this issue more closely, we computed an additional time series $N_{\text {future }}(t, s)$ consisting of the number of avalanches with $t_{i} \in[t, t+s)$. The Spearman correlation coefficient $r_{s}(s)=$ $\operatorname{cor}\left(N(t, s), N_{\text {future }}(t, s)\right)$ then provides an indication whether future avalanche activity correlated with past activity.

For the avalanche catalog OBS, the correlation coefficient $r_{s}(s)$ was relatively low and mostly not significant $(p>0.05)$. Nevertheless, $r_{s}(s)$ exhibited two significant maxima at 24 and 96 hours (black solid line in Figure 11). A very similar pattern was also present for $\mathrm{OBS}_{\text {wet }}$ with overall larger and significant $r_{s}(s)$ values (red solid line in Figure 11). These results highlight once more that during our study period, the majority of avalanches consisted of wet-snow avalanches. For dry-snow avalanches, on the other hand, $r_{s}(s)$ values were lowest, not significant and the trend was different: $r_{s}(s)$ increased up to 72 hours and decreased thereafter (blue solid line in Figure 11).

The correlation coefficients $r_{s}(s)$ for the combined seismic catalog SEIS, as well as the SEIS $_{\text {winter }}$ and SEIS $_{\text {spring }}$ catalogues were generally larger and statistically significant (black, blue and red dotted lines in Figure 11). Nevertheless, trends $r_{s}(s)$ observed in SEIS $_{\text {winter }}$ and SEIS spring $_{\text {showed some sim- }}$ ilarities with those observed in $\mathrm{OBS}_{\text {dry }}$ and $\mathrm{OBS}_{\text {wet }}$. Despite some differences in the exact location of local minima and maxima and, of course, the value of the correlation coefficient, the overall trends in $r_{s}(s)$ exhibited similar features (compare blue and red dotted lines with blue and red solid lines in Figure 11). Indeed, $r_{s}(s)$ exhibited a local maximum at 24 hours and local minima at 72 and 168 hours for SEIS $_{\text {spring }}$ (red dotted lines in Figure 11). For $\mathrm{SEIS}_{\text {winter, }}$ on the other hand, $r_{s}(s)$ reached its maximum at 96 hours 
and decreased thereafter.

The increased temporal resolution obtained through seismic monitoring allowed us to investigate $r_{s}(s)$ in greater detail, especially for shorter time windows ( $s<24$ hours), which is not possible with conventional daily avalanche observations. At these shorter time scales, substantial differences were observed for SEIS $_{\text {winter }}$ and SEIS $_{\text {spring. }}$. While $r_{s}(s)$ generally increased

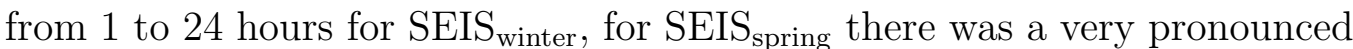
local minimum at 12 hours and the strongest correlation was observed for the shortest time scale of 1 hour. Overall, these results suggest that different processes with different characteristic time scales were responsible for the triggering of dry and wet-snow avalanches in our avalanche catalogues, in line with the results shown in Figure 7 and 8. Avalanche activity in spring closely relates to air temperature and incoming shortwave radiation (e.g. Mitterer and Schweizer, 2013; Helbig et al., 2015), both of which exhibit strong diurnal trends. Avalanche activity in winter, on the other hand, typically relates to precipitation (i.e. storm cycles) (e.g. van Herwijnen and Schweizer, 2011a), for which the normalized covariance exhibited a secondary maximum around 70 hours (not shown), consistent with the long-term correlations observed in Figure 11.

\subsection{Simple avalanche forecasting model}

The results shown in Figure 11 suggest that past dry-snow avalanche activity is only indicative of future avalanche activity when considering large time windows, typically on the order of 3 to 4 days. For wet-snow avalanches, on the other hand, past avalanche activity is only indicative of future avalanche activity for much shorter time windows, either 1 to 3 hours or around 24 
hours. From an avalanche forecasting perspective, this suggests that using past avalanche activity to make predictions about future avalanches in spring will only be reliable for short time scales on the order of hours, while in winter reliability should increase for time scales longer than about 48 hours.

To verify this hypothesis, we evaluated a simple model based on past avalanche activity to predict future avalanches. The model is very similar to the model used by Grasso and Zaliapin (2004) and Schmid et al. (2012) for predicting volcanic eruptions, and is based on the detection of a threshold value on an hourly avalanche activity function $A(t, \Delta)$ (Figure 12), i. e. we want to forecast avalanches on an hourly basis. When an avalanche is detected at time $t_{i}, A(t, \Delta)$ is raised by 1 for a duration $\Delta$ :

$$
A(t, \Delta)=A(t, \Delta)+1 \text { for } t \in\left(t_{i}, t_{i}+\Delta\right] .
$$

An alarm is triggered when $A(t, \Delta)$ exceeds a predefined threshold value $A_{0}$. Thus, the model has two tunable parameters, namely the alarm duration $\Delta$ and the avalanche activity threshold $A_{0}$. Prediction is correct if an avalanche occurs at hour $t$ while $A(t, \Delta)>A_{0}$ (green arrows and dots in Figure 12). However, all other hours with $A(t, \Delta)>A_{0}$ are still considered false alarms (red dots in Figure 12).

We evaluated model performance on the SEIS $_{\text {winter }}$ and SEIS spring catalogues. For $\Delta=24$ hours, the predictive skills of our forecasting model are illustrated in Figure 13. Clearly, for an alarm duration of 24 hours, the model is more accurate in spring than in winter, as reflected by the higher AUC value. For the optimal avalanche activity threshold (1 avalanche in winter and 5 in spring; dots in Figure 13), the POD, FAR and DUR values were also poorer in winter $(0.64,0.96$ and 0.47 , respectively) than in spring $(0.71$, 
0.80 and 0.27 , respectively).

Overall, model performance was better in spring than in winter for the entire range of alarm durations $\Delta$ in terms of model score $S$ and for most of the individual model performance measures (Figure 14). During winter, model performance somewhat improved with alarm duration. For $\Delta>24$ hours, the model resulted in reasonable POD values between 0.6 and 0.7, but very high FAR values of about 0.95 (solid lines in Figure 14b). The improvement of the overall model score $S$ for larger $\Delta$ in winter is mostly attributed to a decrease in the total alarm duration (DUR; black solid line in Figure 14b). During spring, model performance decreased with increasing $\Delta$, predominantly due to increases in FAR and DUR. However, for $\Delta$ between 24 and 48 hours, model performance briefly improved, with typical POD and FAR values of around 0.7 and 0.8 , respectively, and DUR values around 0.2 . This quantitative model performance evaluation shows that based on past avalanche activity, avalanches in spring can more accurately be forecasted than avalanches in winter. Furthermore, it confirms our hypothesis that past wet-snow avalanche activity is indicative of future avalanches for time scales of a day or less, while for dry-snow avalanches this is only the case for longer time scales.

There is a clear link between model performance and the time scales identified in the avalanche catalogues (Sections 3.1 and 3.3). In winter, avalanche activity was clustered for $t_{r}>53$ hours $\left(\right.$ SEIS $_{\text {winter }}$ in Table 1), and model performance generally improved for time scales larger than $t_{r}$. In spring, on the other hand, avalanche activity was clustered for $t_{r}>10$ hours $\left(S E I S_{\text {spring }}\right.$ in Table 1), while our forecasting model performed best for time scales shorter 
than $t_{r}$. While these results seem contradictory, they can be explained based on the correlation analysis of past and future avalanche activity (Figure 11). Indeed, in winter past and future avalanche activity were mostly uncorrelated for time scales shorter than $t_{r}$ (blue dashed line in Figure 11), suggesting that past avalanche activity can only be used for predictions over long time scales $\left(t>t_{r}\right)$. In spring, the correlation of past and future avalanche activity was strongest for time scales shorter than $t_{r}$ and for time scales around 24 hours, while for even longer time scales the correlation decreased (red dashed line in Figure 11). This suggests that avalanche prediction in spring based on past avalanche activity is only effective for shorter time scales $\left(t<t_{r}\right)$, in line with the results of our forecasting model (Figure 14).

To the best of our knowledge there are no other published studies in which an attempt was made to automatically forecast avalanches based on past avalanche activity. In most studies almost exclusively meteorological parameters were used as input (e.g. Brabec and Meister, 2001). However, Schweizer and Föhn (1996) considered snow instability information, and Schirmer et al. (2009) achieved the best results with a nearest neighbor method which used the avalanche danger level of the previous day as additional input. For local forecasting, for instance, Schweizer et al. (2009) used critical new snow depths measured at two different elevations to forecast large dry-snow avalanches in a single avalanche path at time scales ranging from 24 to 120 hours. While their model worked well in terms of POD, with values mostly above 0.7 , the FAR values were generally very high, typically above 0.9 . With regard to wet-snow avalanches, Mitterer and Schweizer (2013) evaluated several models with a variety of meteorological input variables to forecast avalanches in 
the region of Davos at time scales ranging from 24 to 72 hours. Model performance was good with POD values around 0.9 and FAR values around 0.3. On the other hand, Helbig et al. (2015) used a relatively simple model based on air temperature and incoming shortwave radiation to forecast wet-snow avalanches over entire Switzerland at time scales of 24 hours, with typical POD values around 0.6 (FAR values were not reported due to the absence of reliable avalanche occurrence data). It is somewhat surprising that POD values of our very simple model based on past avalanche activity is comparable to more sophisticated models, both in winter and in spring. This may partly be attributed to the fact that we use data that are directly related to instability, whereas most other models use meteorological data which only provide an indirect indication of snow instability (e.g. LaChapelle, 1980; McClung, 2002). While our FAR values are much higher, we would like to point out that since we are using an hourly alarm function, the obtained FAR values are very conservative (see Figure 12). Finally, by including the error diagram in our analysis we have included the total alarm duration in our analysis, which thus far has not been done for avalanche forecasting.

\section{Summary and outlook}

By performing a visual inspection of the waveform and the spectrogram of seismic data recorded in avalanche starting zones, we obtained avalanche catalogues for two entire winters (2010 and 2011) and one period of 10 days with intense wet-snow avalanche activity in the spring of 2013. These avalanche catalogues, consisting of the release times of each avalanche found in the seismic data, were used to investigate clustering and periodicity in avalanche 
activity, and to perform a detailed investigation of how such avalanche activity data could be used for local avalanche forecasting (at spatial scales of $2 \mathrm{~km}^{2}$ ) at time scale of up to 7 days.

Avalanches were strongly clustered in time. The waiting time between avalanches departed from a Poisson process for waiting times larger than $t_{r}$, ranging from 9 to 107 hours. Avalanche activity also exhibited some periodicity, in particular during spring when the covariance of avalanche activity displayed a clear diurnal trend. This comes as no surprise, since it is well known that in spring avalanches typically release during the day, when the energy input into the snow cover is greatest.

Observed seismic avalanche activity compared fairly well with conventional visual avalanche observations for the region of Davos, a region of about $175 \mathrm{~km}^{2}$. This was especially true for wet-snow avalanches, for which all the major regional wet-snow avalanche periods were well captured through seismic monitoring. Dry-snow avalanches, on the other hand, were less frequent in our seismic avalanche catalogues, and most dry-snow avalanche cycles were not well captured through seismic monitoring. These results suggest that monitoring over a larger area by deploying several seismic stations is likely required to provide robust estimates of regional dry-snow avalanche activity.

Since past avalanche activity is often used in avalanche forecasting, we explored the suitability of highly resolved avalanche occurrence data for forecasting purposes. First, we showed that depending on the considered time scale, past avalanche activity is correlated with future avalanche activity. During winter, i.e. for dry-snow avalanches, only long-term correlations were 
observed for time scales larger than 3 days, likely related to the frequency of precipitation events. During spring, on the other hand, short-term correlations were observed, typically for time scales less than 3 hours or around 24 hours, likely related to diurnal patterns in energy input into the snow cover (e.g. air temperature and incoming shortwave radiation).

Based on these results, we evaluated the performance of a simple model based on past avalanche activity to predict future avalanches, similar to models used for predicting volcanic eruptions (e.g. Grasso and Zaliapin, 2004; Schmid et al., 2012). Model performance was better in spring than in winter, especially for very short time scale of up to three hours, and for time scales around 24 hours. For time scales of several days, model performance somewhat improved in winter. While we are not aware of any previous attempts to automatically forecast avalanches based on past avalanche activity, the probability of detection of our very simple model was comparable to the performance of much more sophisticated models based on meteorological input variables (Schweizer et al., 2009; Mitterer and Schweizer, 2013; Helbig et al., 2015).

This work is a first attempt to evaluate the predictive power of past avalanche activity for avalanche forecasting in a quantitative manner. By evaluating model performance in terms of probability of detection, false alarm ratio and total alarm duration, our results suggest that using avalanche activity for avalanche forecasting is more effective in spring than in winter. Future model improvements will include scaling the avalanche activity function with seismic signal duration, since signal duration can serve as a proxy for avalanche size (van Herwijnen et al., 2013; van Herwijnen and Schweizer, 
2011b). Our analysis was based on visual inspection of the seismic data, which is a time consuming endeavor. For operational avalanche forecasting, automatic avalanche detection is therefore required (e.g. Rubin et al., 2012), especially when focusing on natural avalanche activity. Nevertheless, the results reported here clearly show that if highly resolved avalanche occurrence data can be obtained in near real-time, this would be extremely useful for avalanche forecasting.

\section{Acknowledgements}

We would like to thank all the people that helped us with maintaining the field equipment during the winter. M. H. was funded by the Swiss National Science Foundation (SNF, grant 200021-149329). Comments by two anonymous reviewers and Samuel Morin greatly improved the content of this work.

\section{References}

Amitrano, D., Grasso, J., Senfaute, G., 2005. Seismic precursory patterns before a cliff collapse and critical point phenomena. Geophys. Res. Lett. $32,41-55$

Bessason, B., Eiriksson, G., Thorarinsson, O., Thorarinsson, A., Einarsson, S., 2007. Automatic detection of avalanches and debris flows by seismic methods. J. Glaciol. 53, 461-472.

Biescas, B., Dufour, F., Furdada, G., Khazaradze, G., Surinach, E., 2003. Frequency content evolution of snow avalanche seismic signals. Surv. Geophys. 24, 447-464. 
Brabec, B., Meister, R., 2001. A nearest-neighbor model for regional avalanche forecasting. Annals of Glaciology 32, 130-134.

Caplan-Auerbach, J., Huggel, C., 2007. Precursory seismicity associated with frequent, large ice avalanches on Iliamna volcano, Alaska, USA. Journal of Glaciology 53, 128-140. doi:10.3189/172756507781833866.

Deparis, J., Jongmans, D., Cotton, F., Baillet, L., Thouvenot, F., Hantz, D., 2008. Analysis of rock-fall and rock-fall avalanche seismograms in the French Alps. B. Seismol. Soc. Am. 98, 1781-1796. doi:10.1785/0120070082.

Eckerstorfer, M., Bhler, Y., Frauenfelder, R., Malnes, E., 2016. Remote sensing of snow avalanches: Recent advances, potential, and limitations. Cold Reg. Sci. Technol. 121, 126 - 140. doi:10.1016/j.coldregions.2015.11.001.

Faillettaz, J., Louchet, F., Grasso, J.R., 2004. Two-threshold model for scaling laws of noninteracting snow avalanches. Phys. Rev. Lett. 93, 208001. doi:10.1103/PhysRevLett.93.208001.

Faillettaz, J., Pralong, A., Funk, M., Deichmann, N., 2008. Evidence of logperiodic oscillations and increasing icequake activity during the breakingoff of large ice masses. J. Glaciol. 54, 725-737.

Gauer, P., Kern, M., Kristensen, K., Lied, K., Rammer, L., Schreiber, H., 2007. On pulsed doppler radar measurements of avalanches and their implication to avalanche dynamics. Cold Regions Science and Technology 50, $55-71$.

Grasso, J., Zaliapin, I., 2004. Predictability of volcano eruption: Lessons 
from a basaltic effusive volcano. Geophysical Research Letters 31. doi:10.1029/2003GL019022.

Gubler, H., 1979. Acoustic emission as an indication of stability decrease in fracture zones of avalanches. J. Glaciol. 22, 186-188.

Harrison, J., 1976. Seismic signals from avalanches. Armstrong and Ives (Eds.), Avalanche release and snow characteristics. Institute of Arctic and Alpine Research, University of Colorado. Occasional Paper No. 19 , 145150.

Helbig, N., van Herwijnen, A., Jonas, T., 2015. Forecasting wet-snow avalanche probability in mountainous terrain. Cold Regions Science and Technology 120. doi:10.1016/j.coldregions.2015.07.001.

van Herwijnen, A., Dreier, L., Bartelt, P., 2013. Towards a basic avalanche characterization based on the generated seismic signal. Proc. 2013 Int. Snow Sci. W., Grenoble, France , 1033-1037.

van Herwijnen, A., Schweizer, J., 2011a. Seismic sensor array for monitoring an avalanche start zone: design, deployment and preliminary results. J. Glaciol. 57, 257-264.

van Herwijnen, A., Schweizer, J., 2011b. Monitoring avalanche activity using a seismic sensor. Cold Reg. Sci. Tech. 69, 165-176.

Ives, J., Harrison, J., Armstrong, R., 1973. Development of methodology for evaluation and prediction of avalanche hazard in the san juan mountain area of southwestern colorado. interim report for period august 1972 - 
august 1973. Institute of Arctic and Alpine Research, Boulder, Colorado, INSTAAR- I 4-06-7 I 55-2 .

Kapil, J., Datt, P., Kumar, A., Singh, K., Kumar, V., Satyawali, P., 2014. Multi-sensor couplers and waveguides for efficient detection of acoustic emission behavior of snow. Cold Regions Science and Technology 101, $1-$ 13. doi:http://dx.doi.org/10.1016/j.coldregions.2014.01.003.

Kogelnig, A., Wyssen, S., Pichler, J., 2012. Artifical release and detection of avlanches: Managing avalanche risk on traffic infrastructure, a case study from Austria, in: International Snow Science Workshop ISSW 2012, Anchorage AK, U.S.A., 16-21 September 2012, pp. 535-540.

Kumar, R., Indrayan, A., 2011. Receiver Operating Characteristic (ROC) Curve for Medical Researchers. Indian Pediatrics 48, 277-287.

LaChapelle, E., 1980. The fundamental processes in conventional avalanche forecasting. J. Glaciol. 26, 75-84.

Lacroix, P., Grasso, J.R., Roulle, J., Giraud, G., Goetz, D., Morin, S., Helmstetter, A., 2012. Monitoring of snow avalanches using a seismic array: Location, speed estimation, and relationships to meteorological variables. J. Geophys. Res. - Earth 117, F01034. doi:10.1029/2011JF002106.

Lockner, D., 1993. The role of acoustic-emission in the study of rock fracture. Int. J. Rock Mech. Min. 30, 883-899.

Marchetti, E., Ripepe, M., Ulivieri, G., Kogelnig, A., 2015. Infrasound array criteria for automatic detection and front velocity estimation of snow 
avalanches: towards a real-time early-warning system. Nat. Hazards Earth Syst. Sci. Discuss. 3, 2709-2737.

Marzocchi, W., Zaccarelli, L., 2006. A quantitative model for the time-size distribution of eruptions. Journal of Geophysical Research-Solid Earth 111. doi:10.1029/2005JB003709.

McClung, D., 2002. The elements of applied forecasting - Part II: The physical issues and the rules of applied avalanche forecasting. Nat. Hazards 26, $131-146$

McClung, D.M., Schaerer, P., 2006. The Avalanche Handbook. The Mountaineers, Seattle, Washington, U.S.A.

Meister, R., 1995. Country-wide avalanche warning in Switzerland, in: Proceedings International Snow Science Workshop, Snowbird, Utah, U.S.A., 30 October-3 November 1994, ISSW 1994 Organizing Committee, Snowbird UT, USA. pp. 58-71.

Michlmayr, G., Cohen, D., Or, D., 2012. Sources and characteristics of acoustic emissions from mechanically stressed geologic granular media a review. Earth-Science Reviews 112, 97 - 114. doi:http://dx.doi.org/10.1016/j.earscirev.2012.02.009

Mitterer, C., Schweizer, J., 2013. Analysis of the snow-atmosphere energy balance during wet-snow instabilities and implications for avalanche prediction. The Cryosphere 7, 205-216.

Molchan, G., 1997. Earthquake prediction as a decision-making problem. Pure and Applied Geophysics 149, 233-247. 
Niemz, P., Brunner, A., Walter, O., 2009. Investigation of the mechanism of failure behaviour of wood based materials using acoustic emission analysis and image processing. Wood Res. 54, 49-62.

Reiweger, I., Mayer, K., Steiner, K., Dual, J., Schweizer, J., 2015. Measuring and localizing acoustic emission events in snow prior to fracture. Cold Regions Science and Technology 110, 160 - 169.

Rosti, J., Koivisto, J., Alava, M.J., 2010. Statistics of acoustic emission in paper fracture: precursors and criticality. J. Stat. Mech. - Theory E. 2010, P02016. doi:10.1088/1742-5468/2010/02/P02016.

Rubin, M., Camp, T., van Herwijnen, A., Schweizer, J., 2012. Automatically detecting avalanche events in passive seismic data. Proc. 11th Int. Conf.Mach. Learn. Appl. 1, 13-20.

Schirmer, M., Lehning, M., Schweizer, J., 2009. Statistical forecasting of regional avalanche danger using simulated snow-cover data. J. Glaciol. 55, 761-768.

Schmid, A., Grasso, J.R., Clarke, D., Ferrazzini, V., Bachelery, P., Staudacher, T., 2012. Eruption forerunners from multiparameter monitoring and application for eruptions time predictability (Piton de la Fournaise). Journal of Geophysical Research-Solid Earth 117. doi:10.1029/2012JB009167.

Schweizer, J., Föhn, P., 1996. Avalanche forecasting - An expert system approach. J. Glaciol. 42, 318-332. 
Schweizer, J., van Herwijnen, A., 2013. Can near real-time avalanche occurrence data improve avalanche forecasting? Proc. 2013 Int. Snow Sci. W., Grenoble, France , 195-198.

Schweizer, J., Kronholm, K., Wiesinger, T., 2003. Verification of regional snowpack stability and avalanche danger. Cold Reg. Sci. Technol. 37, 277288.

Schweizer, J., Mitterer, C., Stoffel, L., 2009. On forecasting large and infrequent snow avalanches. Cold Regions Science and Technology 59, $234-$ 241.

Scott, E., Hayward, C., Kubichek, R., Hamann, J., Pierre, J., Comey, B., Mendenhall, T., 2007. Single and multiple sensor identification of avalanche-generated infrasound. Cold Reg. Sci. Technol. 47, 159-170.

Spillmann, T., Maurer, H., Green, A., Heincke, B., Willenberg, H., Husen, S., 2007. Microseismic investigation of an unstable mountain slope in the Swiss Alps. J. Geophys. Res. - Sol. Ea. 112. doi:10.1029/2006JB004723.

Suriñach, E., Furdada, G., Sabot, F., Biescas, B., Vilaplana, J., 2001. On the characterization of seismic signals generated by snow avalanches for monitoring purposes. Ann. Glaciol. 32(7), 268-274. doi:doi:10.3189/172756401781819634.

Suriñach, E., Vilajosana, I., Khazaradze, G., Biescas, B., Furdada, G., Vilaplana, J., 2005. Seismic detection and characterization of landslides and other mass movements. Nat. Hazards Earth Syst. Sci. 5(6), 791-798. 
Talbi, A., Nanjo, K., Zhuang, J., Satake, K., Hamdache, M., 2013. Interevent times in a new alarm-based earthquake forecasting model. Geophysical Journal International 194, 1823-1835. doi:10.1093/gji/ggt194.

Tatard, L., Grasso, J.R., Helmstetter, A., Garambois, S., 2010. Characterization and comparison of landslide triggering in different tectonic and climatic settings. Journal of Geophysical Research-Earth Surface 115. doi:10.1029/2009JF001624.

Thüring, T., Schoch, M., van Herwijnen, A., Schweizer, J., 2015. Robust snow avalanche detection using supervised machine learning with infrasonic sensor arrays. Cold Regions Science and Technology 111, 60 - 66 .

Wilks, D., 2011. Statistical methods in the atmospheric sciences. volume 100 of International Geophysics Series. 3rd ed., Academic Press, San Diego CA, U.S.A.

Woo, S., Choi, N., Cho, N., 2008. Characterization of the fracture process of notched glass fiber/aluminum hybrid laminates by acoustic emission. Comps. Sci. Technol. 68, 1521-1530. doi:10.1016/j.compscitech.2007.10.020. 
Table 1: Overview of the total number of avalanches $N$, the coefficient of variation $\eta$ of the waiting time, and the threshold time $t_{r}$ for the avalanche catalogues.

\begin{tabular}{l|ccc} 
catalog & $N$ & $\eta$ & $t_{r}$ (hours) \\
\hline WAN2010 & 385 & 2.3 & 11 \\
WAN2010 $_{\text {winter }}$ & 58 & 0.95 & 107 \\
WAN2010 $_{\text {spring }}$ & 327 & 3.1 & 8 \\
WAN2011 & 154 & 2.5 & 42 \\
WAN2011 $_{\text {winter }}$ & 88 & 2.3 & 55 \\
WAN2011 $_{\text {spring }}$ & 66 & 2.1 & 25 \\
DFB2013 $^{\text {SEIS }}$ & 52 & 1.9 & 12 \\
SEIS $_{\text {spring }}$ & 146 & 1.9 & 53 \\
\hline
\end{tabular}


Table 2: Correlation analysis between daily regional avalanche activity recorded through conventional visual observations and daily avalanche activity recorded through seismic monitoring. The Spearman correlation coefficient $r_{s}$ as well as the significance level $p$ are shown.

\begin{tabular}{l|cc|cc|cc}
\hline & \multicolumn{2}{|c|}{ OBS } & \multicolumn{2}{c|}{ OBS $_{\text {dry }}$} & \multicolumn{2}{c}{ OBS $_{\text {wet }}$} \\
\hline catalog & $r_{s}$ & $p$ & $r_{s}$ & $p$ & $r_{s}$ & $p$ \\
\hline WAN2010 & 0.26 & $8 \times 10^{-3}$ & -0.18 & 0.06 & 0.28 & $3 \times 10^{-3}$ \\
WAN2011 & 0.37 & $<10^{-3}$ & 0.11 & 0.21 & 0.35 & $<10^{-3}$ \\
DFB2013 & 0.58 & 0.06 & -0.04 & 0.90 & 0.42 & 0.19 \\
\hline
\end{tabular}


Table 3: Correlation analysis between avalanche danger rating and previous day avalanche activity. The median daily avalanche activity as well as the interquartile range (in brackets) are shown for each danger level as well as the Spearman correlation coefficient $r_{s}$ and the significance level $p$.

\begin{tabular}{l|ccccc|cccccc|cccc}
\hline & \multicolumn{4}{|c|}{ Danger rating all } & \multicolumn{4}{c|}{ Danger rating dry } & \multicolumn{4}{c}{ Danger rating wet } \\
\hline Catalog & 1 & 2 & 3 & $r_{s}$ & $p$ & 1 & 2 & 3 & $r_{s}$ & $p$ & 2 & 3 & $r_{s}$ & $p$ \\
\hline OBS & $0(0)$ & $0(3)$ & $4(20)$ & 0.29 & $<10^{-3}$ & $0(0)$ & $0(0)$ & $2(14)$ & 0.33 & $<10^{-3}$ & $0(0)$ & $0.5(13)$ & 0.27 & 0.04 \\
SEIS & $1(2)$ & $0(1)$ & $1(3)$ & 0.19 & $<10^{-3}$ & $1(1)$ & $1(3)$ & $1(2)$ & 0.06 & 0.36 & $1(4)$ & $3(8)$ & 0.24 & 0.05 \\
\hline
\end{tabular}



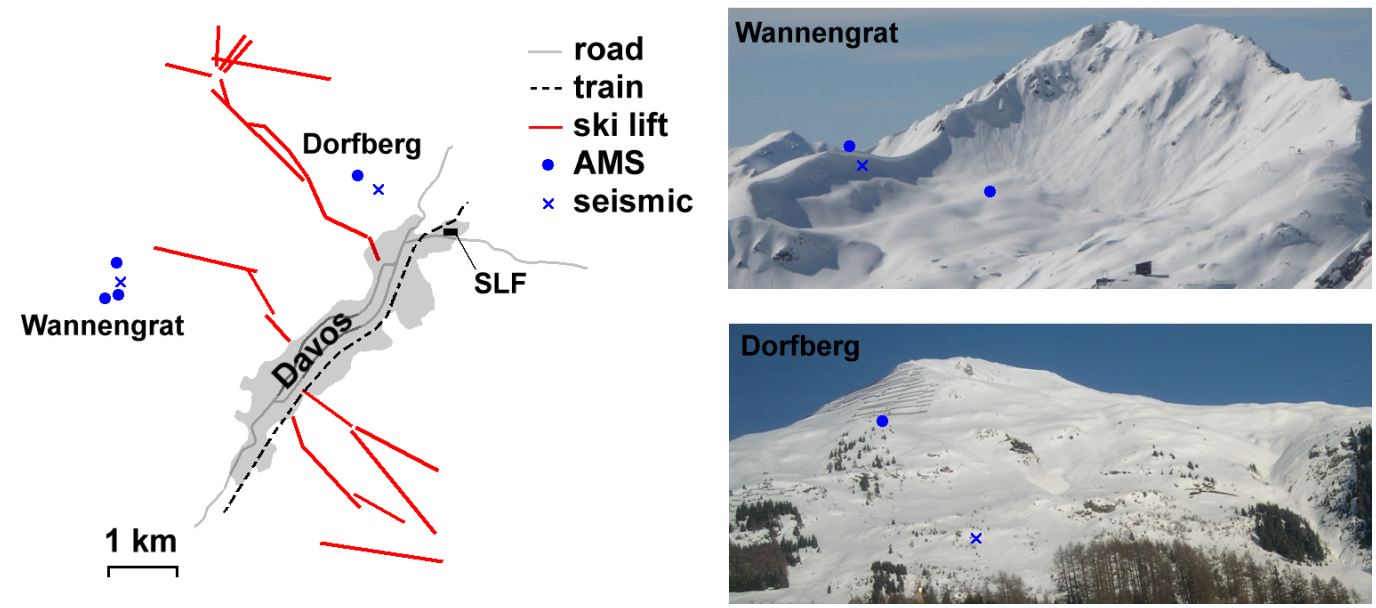

Figure 1: Overview of the field sites instrumented with seismic monitoring systems around Davos, Switzerland. Also shown are the main roads, railway line, mountain cable ways and the locating of automatic meteorological stations (AMS). 
a
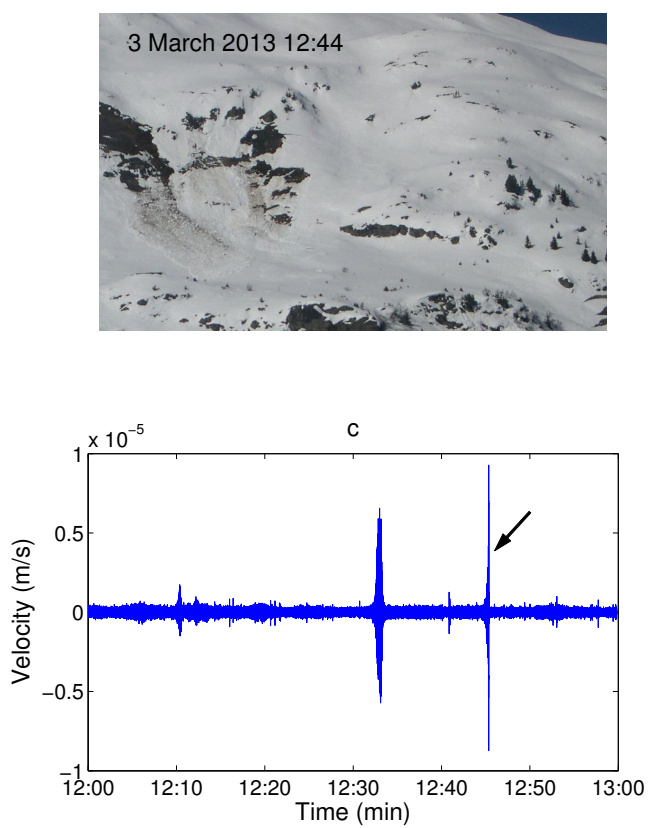

b

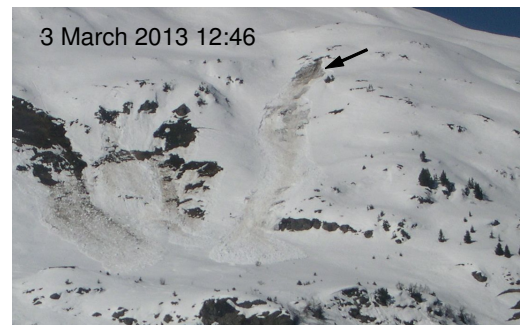

d

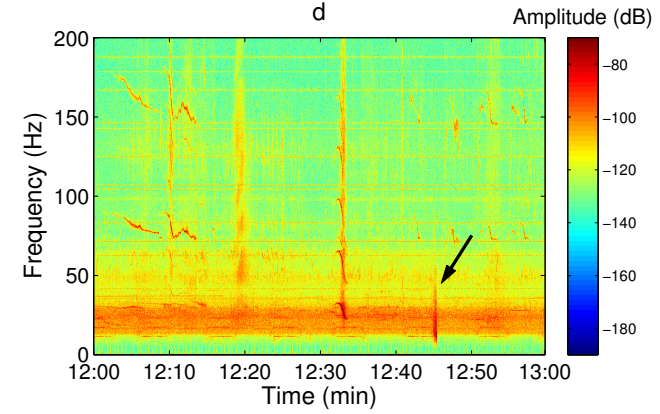

Figure 2: (a,b) Images from the automatic camera at DFB taken on 3 March 2013 at 12:44 and 12:46 showing the release of an avalanche (arrow). (c) Seismic waveform recorded at DFB on 3 March 2013 between 12:00 and 13:00. The arrow shows the signal generated by the avalanche. (d) Corresponding spectrogram showing the frequency content of the signal with time. The black arrow shows the signal generated by the avalanche. 
a
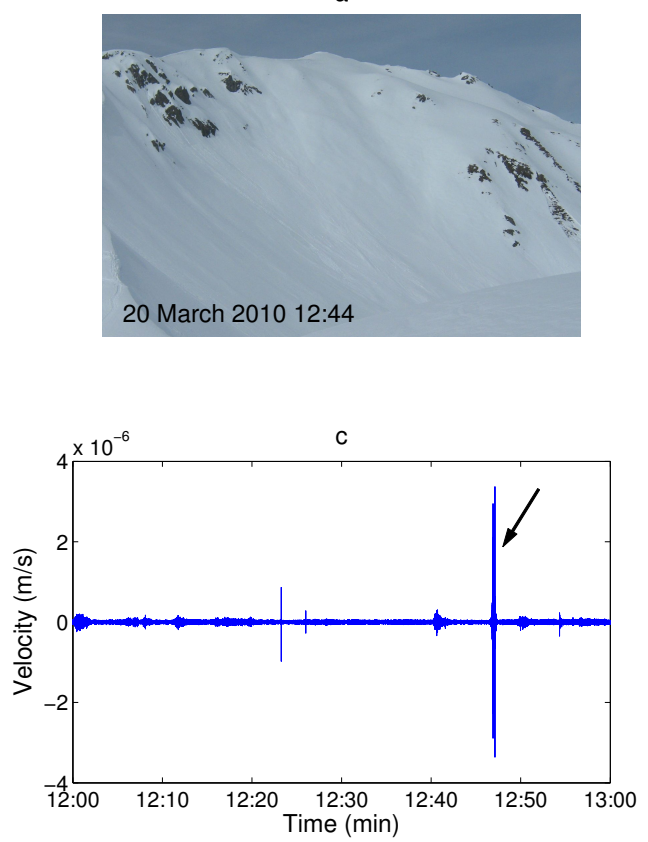

b

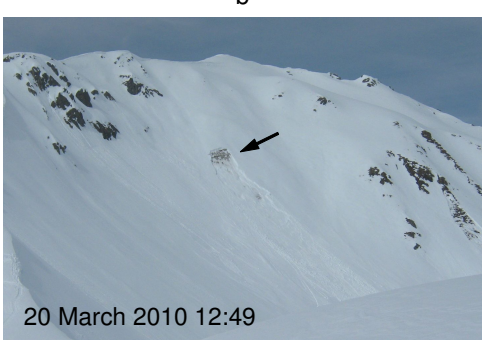

d

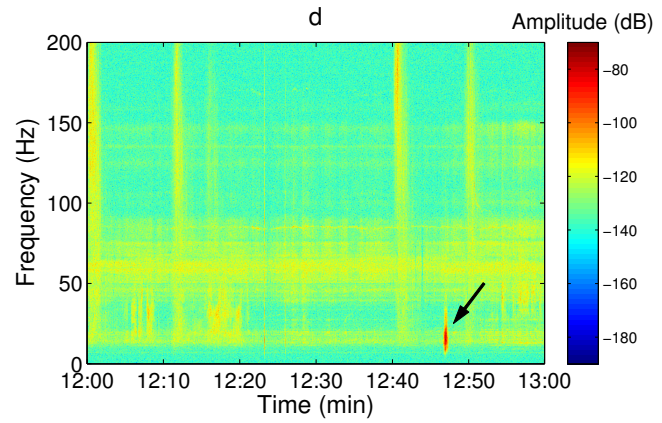

Figure 3: (a,b) Images from the automatic camera at WAN taken on 20 March 2010 at 12:44 and 12:48 showing the release of an avalanche (arrow). (c) Seismic waveform recorded at WAN on 20 March 2010 between 12:00 and 13:00. The arrow shows the signal generated by the avalanche. (d) Corresponding spectrogram showing the frequency content of the signal with time. The black arrow shows the signal generated by the avalanche. 


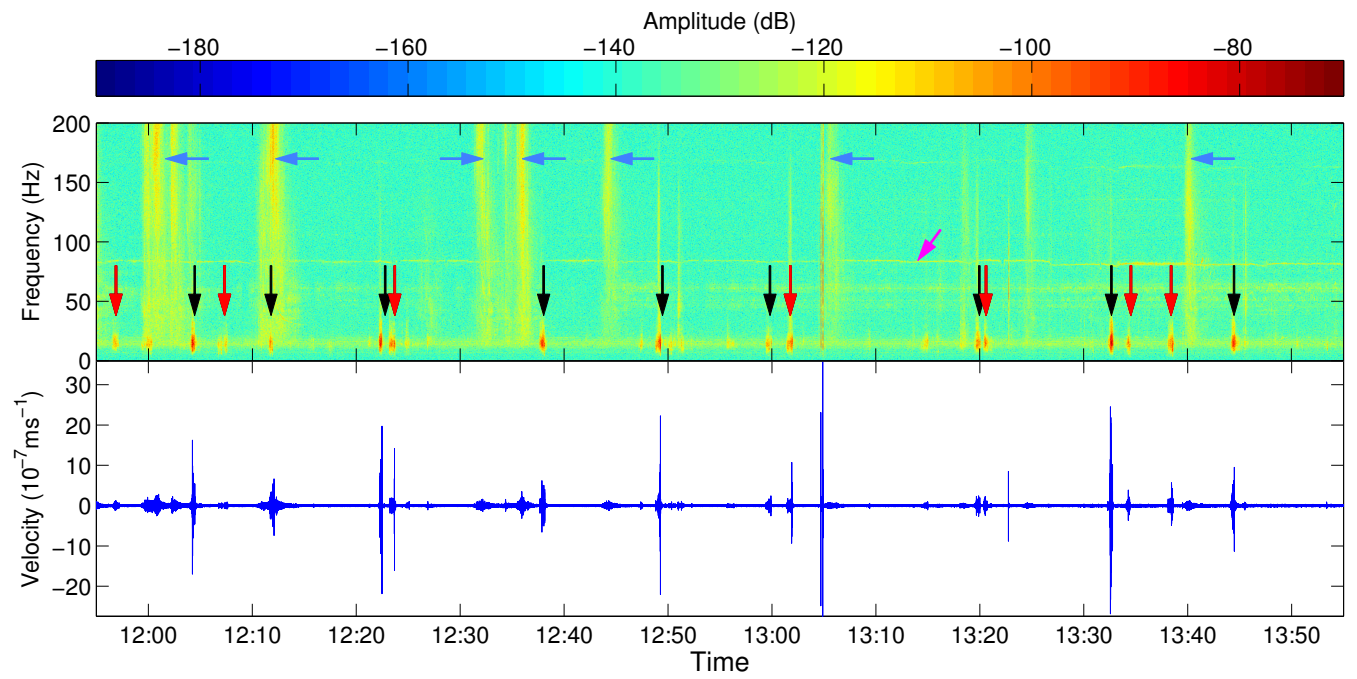

Figure 4: Seismic signals generated by avalanches and environmental noise for two hours on 23 March 2010. Top: spectrogram showing the frequency content of the signal with time. The black arrows show confirmed avalanches, based on images from automatic cameras. The red arrows show visually picked avalanches without confirmation. The blue arrows show airplane signals. The magenta arrow shows the noise band from ski lifts. Bottom: corresponding waveform. 


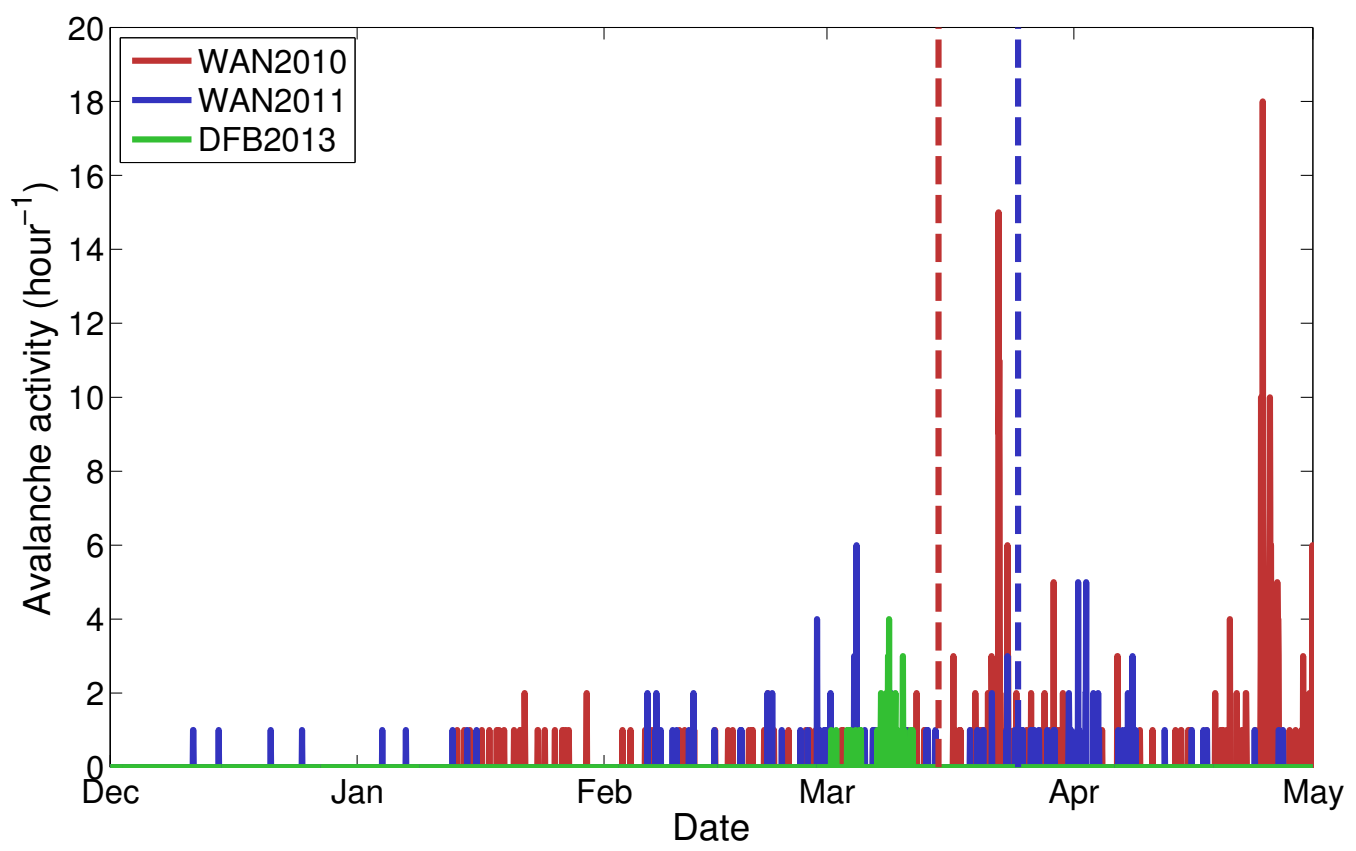

Figure 5: Hourly avalanche activity observed through seismic monitoring for WAN2010 (red line), WAN2011 (blue line), and DFB2013 (green line). The cutoff date between winter and spring for WAN2010 was 15 March 2010 (red dashed line) and for WAN2011 it was 25 March 2011 (blue dashed line). 


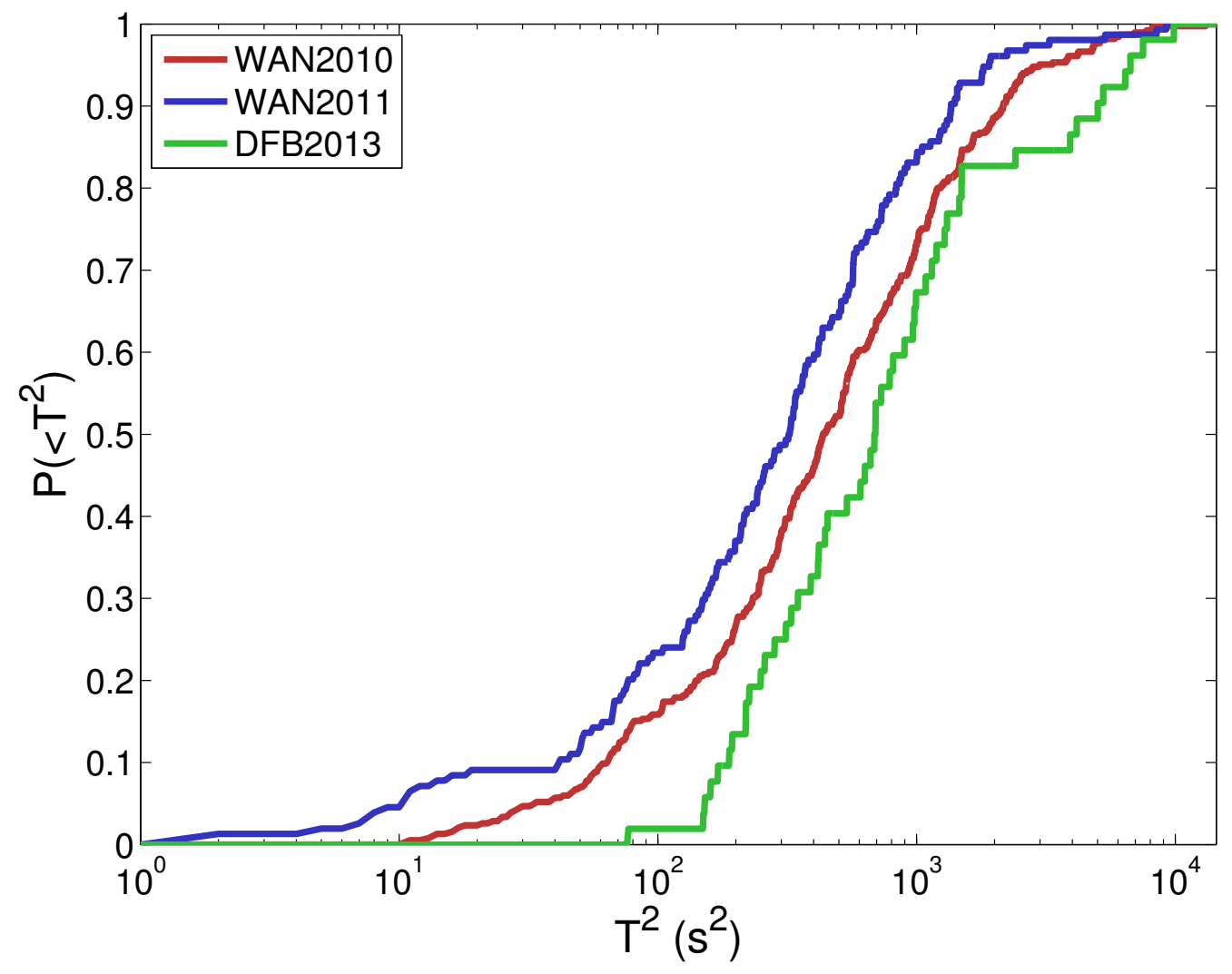

Figure 6: Cumulative distribution of the square of seismic signal duration $T$ for WAN2010 (red line), WAN2011 (blue line), and DFB2013 (green line). 

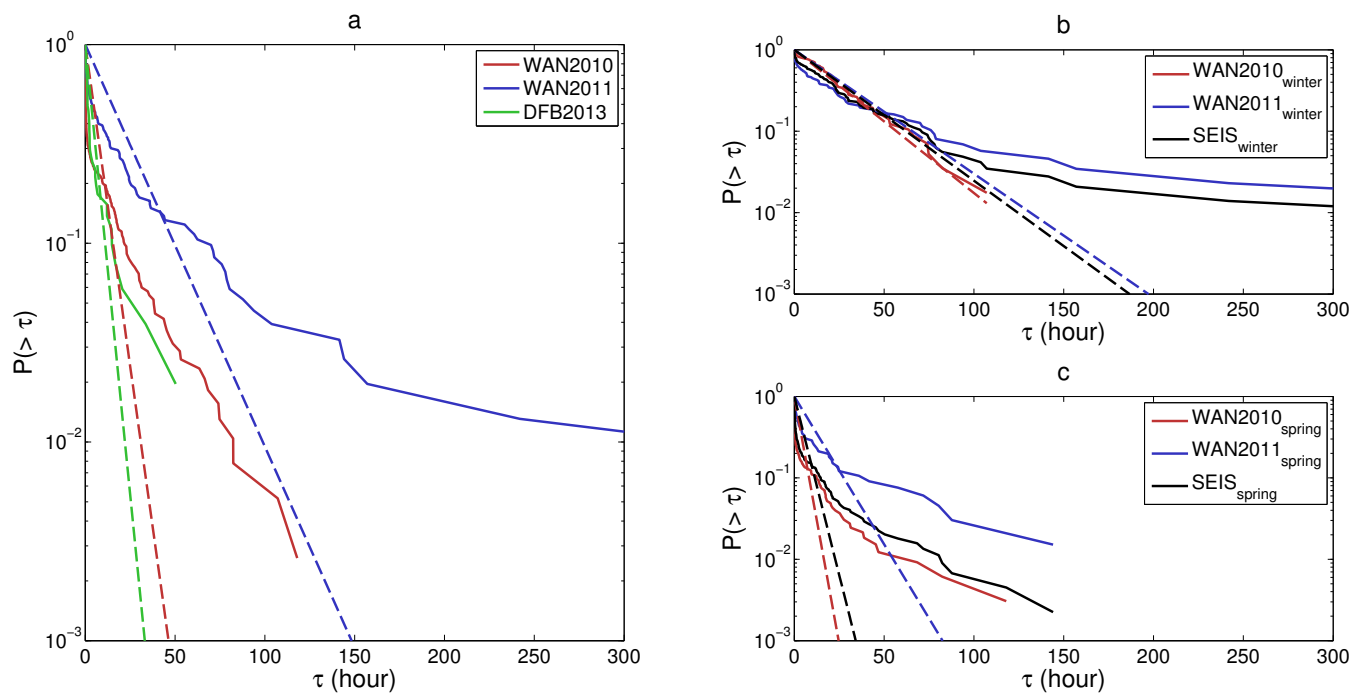

Figure 7: Cumulative distribution of avalanche waiting time $\tau$. The dashed lines represent the corresponding exponential distribution computed for each catalog. (a) For WAN2010 (red line), WAN2011 (blue line), and DFB2013 (green line). (b) For WAN2010 winter (red line), WAN2011 winter $_{\text {(blue line) and SEIS }}$ winter (black line). (c) For WAN2010 spring $_{\text {(red }}$ line), WAN2011 $1_{\text {spring }}$ (blue line) and SEIS spring (black line). 

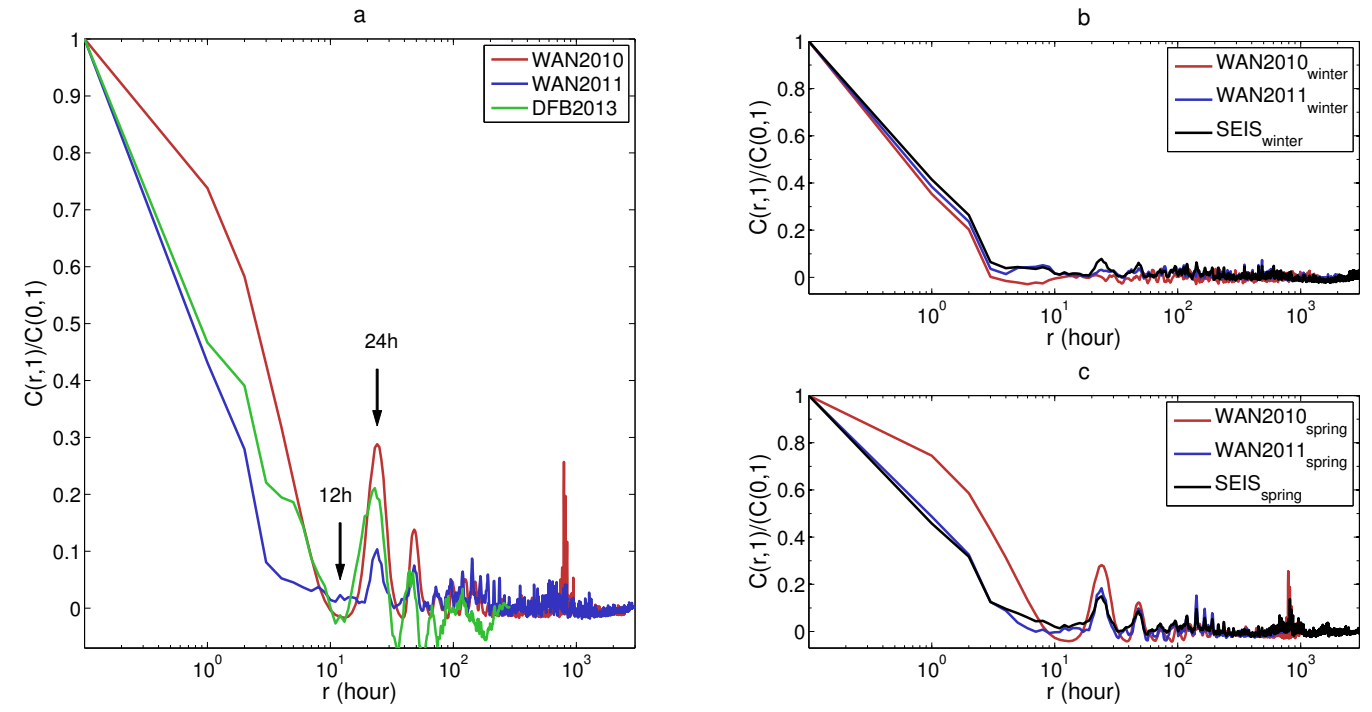

Figure 8: Normalized covariance $C(r, 1) / C(0,1)$ of hourly avalanche activity with lag time $r$. (a) For WAN2010 (red line), WAN2011 (blue line), and DFB2013 (green line). (b) For

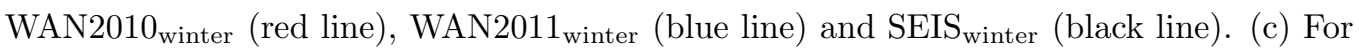

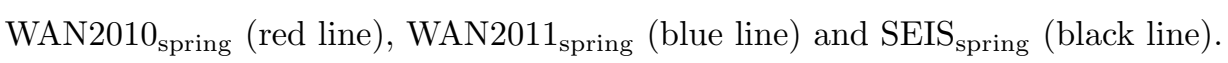




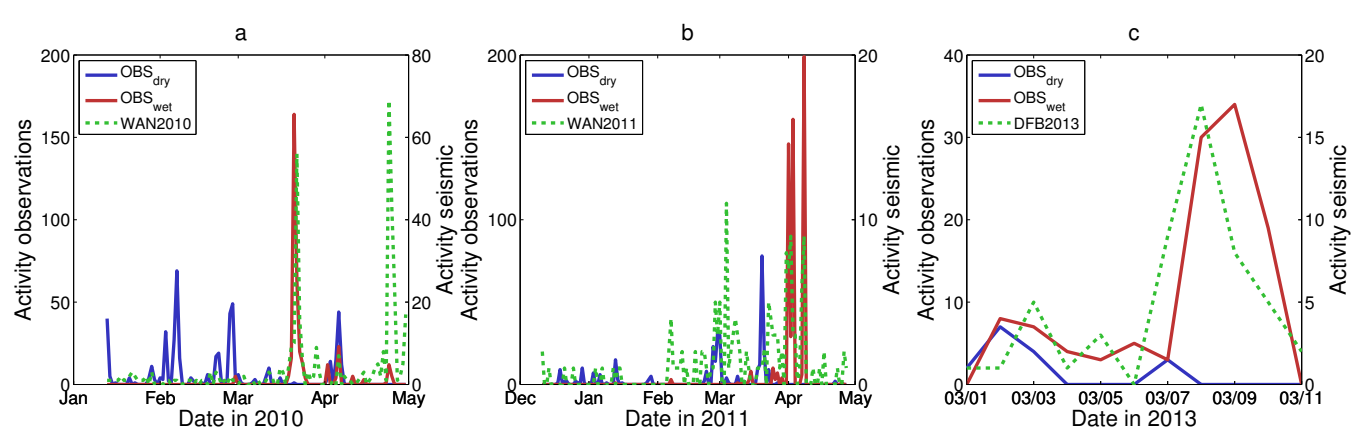

Figure 9: Comparison of dry-snow (blue line) and wet-snow (red line) daily avalanche activity observed through conventional visual observations in the region of Davos with avalanche activity observed through seismic monitoring (green dashed lines) for the three avalanche catalogues (a) WAN2010, (b) WAN2011, (c) DFB2013. 

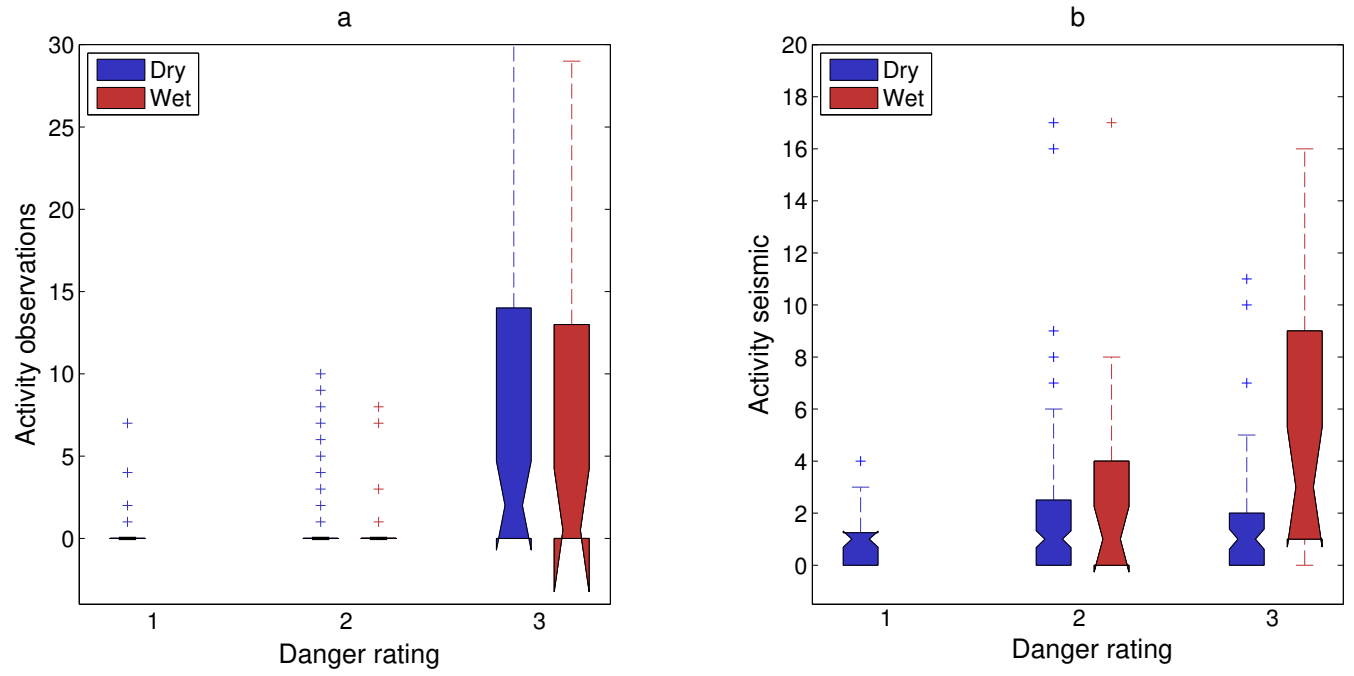

Figure 10: (a) Avalanche activity observed through conventional visual observations with forecasted dry-snow (blue) and wet-snow (red) avalanche danger rating for the region of Davos. (b) Same for avalanche activity observed through seismic monitoring. On each box, the notch shows the location of the median, the edges of the box are the 1st and 3rd quartiles, the whiskers show the range of observed values that fall within 1.5 times the interquartile range above and below the interquartile range, and the crosses show outliers. 


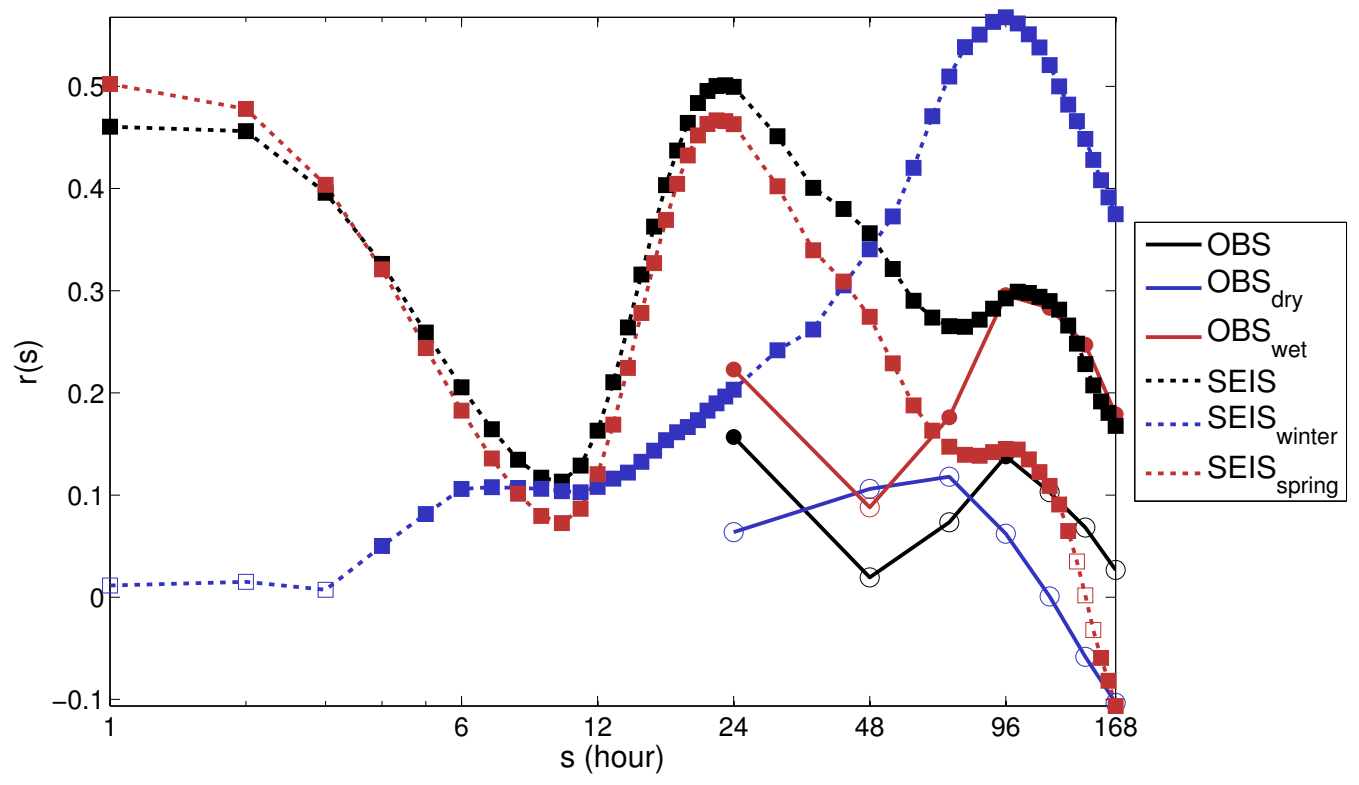

Figure 11: Correlation coefficient $r_{s}(s)$ between past and future avalanche activity with windows length $s . r_{s}(s)$ was computed for the OBS avalanche catalogues (circles and solid lines with colors as indicated in the legend) as well as for the SEIS avalanche catalogues (squares and dotted lines with colors as indicated in the legend). Filled symbols indicate correlation coefficients which were significant $(p \leq 0.05)$, while open symbols indicate correlation coefficients which were not significant $(p>0.05)$ 


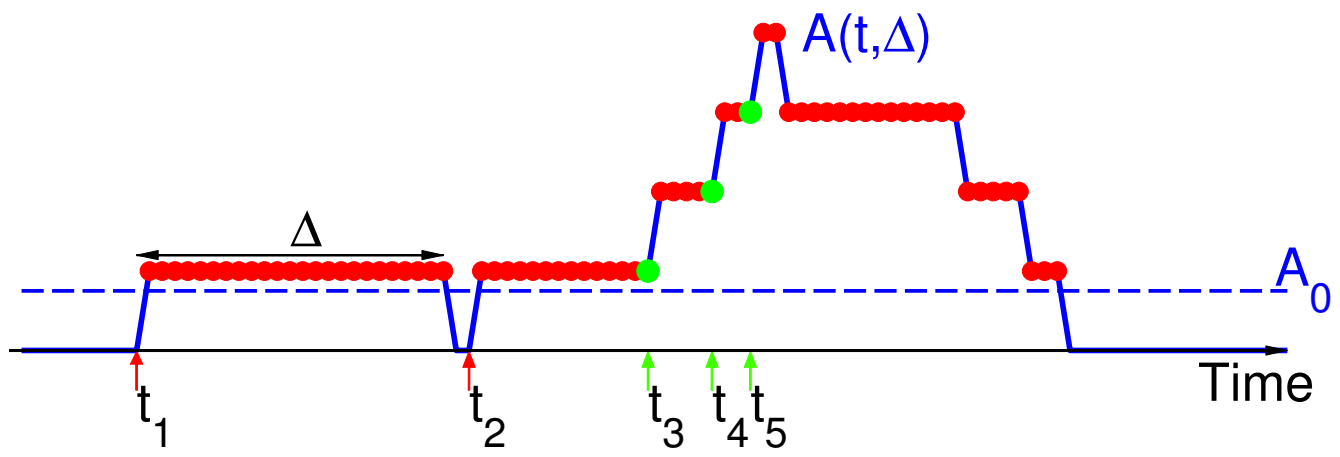

Figure 12: Simple model to predict avalanches based on the avalanche activity function $A(t, \Delta)$ (blue line). The tunable parameters are the alarm duration $\Delta$ and the avalanche activity threshold $A_{0}$ (blue dashed line). The arrows indicate avalanche events; red arrows are missed events, green arrows are correctly predicted events. The red open circles depict false alarms and the green open circles correct alarms. 
a

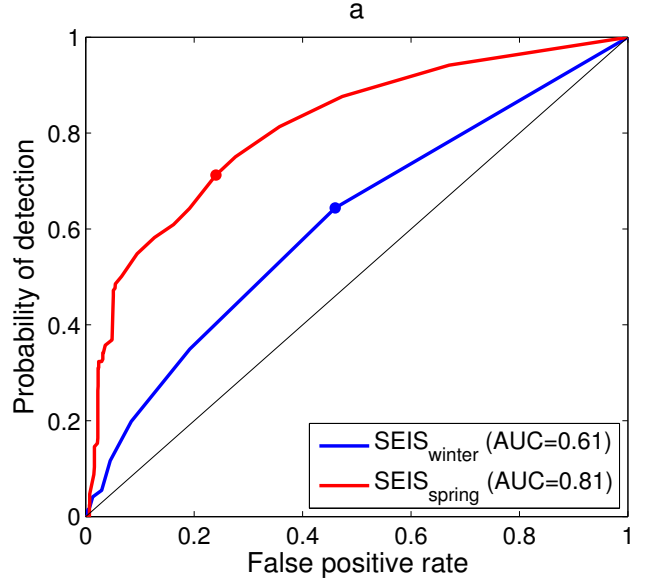

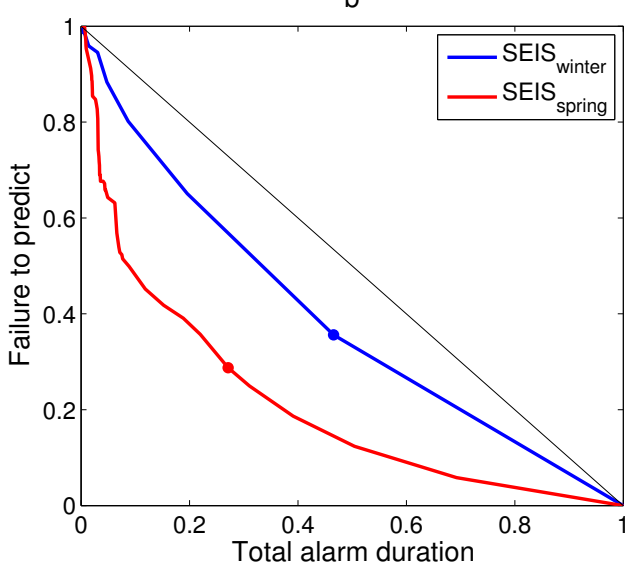

Figure 13: (a) ROC curve for an alarm duration $\Delta$ of 24 hours for the $\operatorname{SEIS}_{\text {winter }}$ (blue) and SEIS spring (red) avalanche catalogues. The black line shows the line of random chance (50-50 chance) and the blue and red dots show the optimal model performance. (b) Error diagram for an alarm duration $\Delta$ of 24 hours for the SEIS $_{\text {winter }}$ (blue) and SEIS spring $_{\text {(red) }}$ avalanche catalogues. The black line shows the line of random chance (50-50 chance) and the blue and red dots show the optimal model performance. 

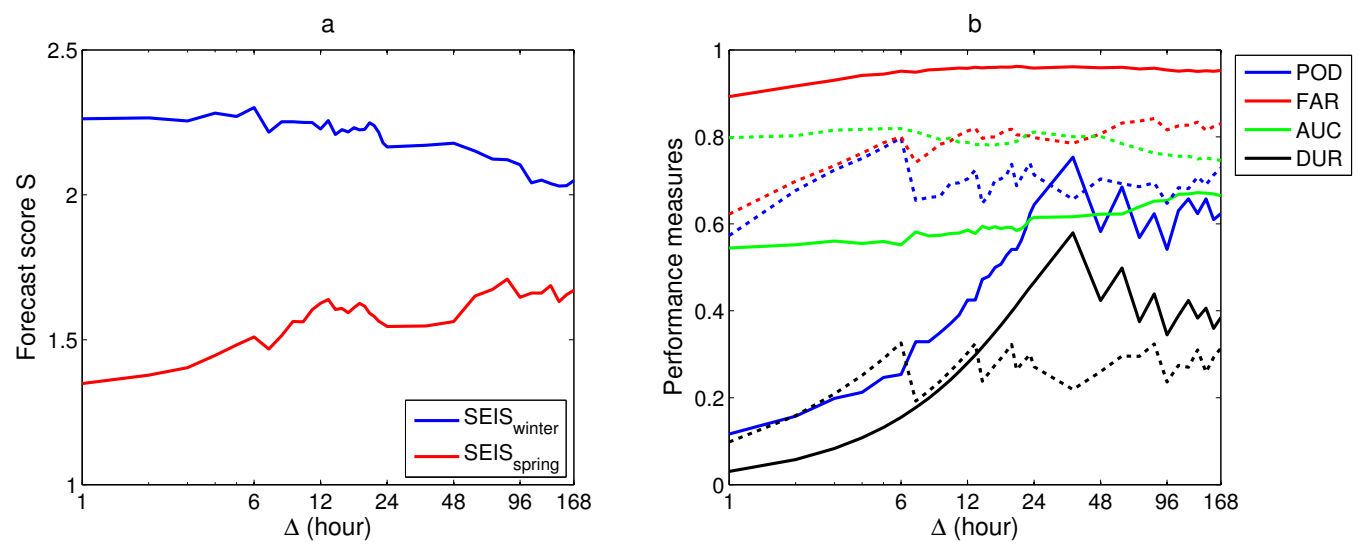

Figure 14: (a) Forecasting score $S$ with alarm duration $\Delta$ for the SEIS winter $_{\text {(blue) and }}$ SEIS $_{\text {spring }}$ (red) avalanche catalogues. (b) Individual performance measures POD (blue), FAR (red), AUC (green) and DUR (black) with alarm duration $\Delta$ for the $\mathrm{SEIS}_{\text {winter }}$ (solid) and SEIS $_{\text {spring }}$ (dashed) avalanche catalogues. 